\title{
Cisplatin, Gemcitabine, and Lapatinib as Neoadjuvant Therapy for Muscle-Invasive Bladder Cancer
}

\section{Vivek Narayan, MD ${ }^{1}$ \\ Ronac Mamtani, MD \\ Stephen Keefe, MD \\ Thomas Guzzo, MD² \\ S. Bruce Malkowicz, MD2 \\ David J. Vaughn, MD'}

${ }^{1}$ Division of Medical Oncology,

Hospital of the University of Pennsylvania,

Abramson Cancer Center, Philadelphia, PA,

${ }^{2}$ Department of Urology,

Hospital of the University of Pennsylvania,

Philadelphia, PA, USA
Correspondence: David J. Vaughn, MD

Department of Medicine,

Division of Hematology/Medical Oncology,

Hospital of the University of Pennsylvania,

7th Floor PCAM South Pavilion, Philadelphia,

PA 19104, USA

Tel: $1-215-349-8140$

Fax: 1-215-662-7804

E-mail: david.vaughn@uphs.upenn.edu

Received October 22, 2015

Accepted November 9, 2015

Published Online December 2, 2015

\section{Purpose}

We sought to investigate the safety and efficacy of gemcitabine, cisplatin, and lapatinib (GCL) as neoadjuvant therapy in patients with muscle-invasive bladder cancer (MIBC) planned for radical cystectomy.

\section{Materials and Methods}

Four cycles of GCL were administered as neoadjuvant therapy for patients with MIBC. Although initially designed as a phase II efficacy study with a primary endpoint of pathologic complete response at the time of radical cystectomy, the dose selected for investigation proved excessively toxic. A total of six patients were enrolled.

\section{Results}

The initial four patients received gemcitabine $1,000 \mathrm{mg} / \mathrm{m}^{2}$ intravenously on days 1 and 8 and cisplatin $70 \mathrm{mg} / \mathrm{m}^{2}$ intravenously on day 1 of each 21-day treatment cycle. Lapatinib was administered as $1,000 \mathrm{mg}$ orally daily starting one week prior to the initiation of cycle 1 of gemcitabine and cisplatin (GC) and continuing until the completion of cycle 4 of GC. These initial doses were poorly tolerated, and the final two enrolled patients received a reduced lapatinib dose of $750 \mathrm{mg}$ orally daily. However, reduction of the lapatinib dose did not result in improved tolerance or drug-delivery, and the trial was terminated early due to excessive toxicity. Grade 3/4 toxicities included diarrhea (33\%), nausea/vomiting (33\%), and thrombocytopenia (33\%).

\section{Conclusion}

The addition of lapatinib to GC as neoadjuvant therapy for MIBC was limited by excessive treatment-related toxicity. These findings highlight the importance of thorough dose-escalation investigation of combination therapies prior to evaluation in the neoadjuvant setting, as well as the limitations of determination of maximum tolerated dose for novel targeted combination regimens.

\section{Introduction}

The definitive management of muscle-invasive bladder cancer (MIBC) has traditionally involved curative-intent radical cystectomy with bilateral pelvic lymph node dissection. [1] In 2003, in a phase III Intergroup study of MIBC patients,
Key words

Urothelial carcinoma, Drug therapy, Molecular targeted therapy, Epidermal growth factor receptor, Cystectomy a significant improvement in overall survival (OS) was demonstrated with the addition of neoadjuvant methotrexate, vinblastine, doxorubicin, and cisplatin (MVAC) chemotherapy to radical cystectomy [2]. A subsequent metaanalysis of over 3,000 patients reported an absolute improvement in 5-year OS of 5\% with the use of platinum-based combination neoadjuvant chemotherapy [3]. Therefore, the 
currently accepted standard of care in surgically-fit patients with MIBC is the use of cisplatin-based neoadjuvant chemotherapy prior to radical cystectomy [4].

In the metastatic setting, similar OS and response rates with an improved toxicity profile have been demonstrated with the regimen of gemcitabine and cisplatin (GC) when compared with conventional-dose MVAC [5]. These findings have commonly been extrapolated to the perioperative setting and have resulted in the frequent use of GC as neoadjuvant chemotherapy. Indeed, a recent survey of U.S. medical oncologists at both academic and community centers found that $90 \%$ offer GC as neoadjuvant chemotherapy for MIBC [6].

Importantly, the survival benefit of neoadjuvant chemotherapy appears to be related to downstaging of the tumor to a complete pathologic response (pT0). For example, in the intergroup trial, neoadjuvant MVAC was associated with a significantly increased pT0 rate ( $38 \%$ vs. $15 \%$ ), and patients who successfully attained a pT0 response achieved a more durable survival benefit (5-year survival rate of $85 \%$ ) [2]. Therefore, novel methods for maximizing the pT0 rate with neoadjuvant therapy are highly desired.

Epidermal growth factor receptors 1 and 2 (EGFR and HER-2) are frequently overexpressed in bladder urothelial carcinomas and have been associated with a poor prognosis. $[7,8]$ Up to $70 \%$ of urothelial carcinomas overexpress EGFR and/or HER-2, and ligand-induced EGFR/HER-2 heterodimerization may trigger potent proliferative and survival signals [7,9]. Therefore, dual-inhibition of EGFR and HER-2 represents an attractive therapeutic strategy for management of urothelial carcinoma.

Lapatinib (Tykerb, GlaxoSmithKline, London, UK) is a small molecule tyrosine kinase inhibitor that targets both the EGFR and HER-2 receptors, thereby resulting in inhibition of downstream effector pathways, growth arrest, and cellular apoptosis $[10,11]$. A preclinical study of lapatinib in combination with GC in human bladder cancer cell lines demonstrated anti-tumor activity with synergistic effects [12]. The suggested dose range for lapatinib in phase II trials is 1,250$1,500 \mathrm{mg}$ daily $[13,14]$, and multiple prior clinical trials of lapatinib in combination with cytotoxic chemotherapy have been conducted with daily lapatinib doses of 1,250-1,500 mg $[15,16]$. In addition, a phase I trial in metastatic bladder cancer of gemcitabine, cisplatin, and lapatinib (GCL) at a dose range of 750-1,250 mg daily reported a maximum tolerated lapatinib dose of $1,250 \mathrm{mg}$ daily [17].

Based on these background findings, we designed a prospective phase II trial to evaluate the safety and efficacy of neoadjuvant GCL in patients with MIBC undergoing radical cystectomy. The selected lapatinib dose was 1,000 mg daily, and the efficacy of GCL treatment was to be assessed by the rate of PT0 response at the time of radical cystectomy.
However, due to excessive therapy-related toxicities at the selected treatment dose, the study was terminated prematurely. Here, we report the study results for eligible patients treated on the neoadjuvant GCL protocol and discuss the difficulties with determination of reliable maximum tolerated dose in the evaluation of novel targeted combination regimens.

\section{Materials and Methods}

\section{Eligibility}

Eligible patients were required to have pathologically-confirmed MIBC after an initial transurethral resection of the bladder tumor (TURBT) and no evidence of nodal or distant metastatic disease (clinical stage T2-T4, N0, M0). An archived tumor specimen from a prior TURBT was required to demonstrate overexpression of EGFR and / or HER-2 by standard immunohistochemistry (IHC) assay (> 1+ expression). Patients were required to have adequate bone marrow, cardiac, hepatic, and renal function, including an estimated creatinine clearance $>60 \mathrm{~mL} / \mathrm{min}$ (determined by CockcroftGault formula calculation) and a baseline left ventricular ejection fraction $\geq 50 \%$ (assessed by multigated acquisition scan or echocardiography). In addition, eligible patients were required to have an Eastern Cooperative Oncology Group performance status of 0-1 and must have been deemed a suitable candidate for radical cystectomy with curative intent. Because lapatinib is a substrate for CYP3A4, the concomitant use of medications that are inducers or inhibitors of CYP3A4 was prohibited.

\section{Study design and treatment plan}

This study was designed as a non-randomized, singleinstitution, open-label study to evaluate the safety and efficacy of four cycles of neoadjuvant GCL. Treatment consisted of gemcitabine $1,000 \mathrm{mg} / \mathrm{m}^{2}$ intravenously on days 1 and 8 and cisplatin $70 \mathrm{mg} / \mathrm{m}^{2}$ intravenously on day 1 of each 21-day treatment cycle. Carboplatin area under the curve 4.5 on day 1 of each treatment cycle could be substituted at the discretion of the treating physician for renal or other toxicity requiring discontinuation of cisplatin. Lapatinib $1,000 \mathrm{mg}$ was administered orally and continuously once daily in the morning and in a fasting state starting on day 7 ( 1 week prior to cycle 1 day 1 of GC) and continuing until cycle 4 day 21 . In cases of drug-related toxicity $\geq$ grade 3 , a one-time dosage reduction of lapatinib to $750 \mathrm{mg}$ once daily was permitted. In addition, a delay in lapatinib treatment 
was permitted for up to 2 weeks to allow for resolution of drug-related toxicities. Prophylactic anti-emetics were administered at the discretion of the treating physician. The protocol-specified management of diarrheal toxicity included the initiation of anti-motility agents every 2-4 hours until resolution of symptoms for at least 12 hours. After completing four cycles of GCL therapy, patients proceeded to radical cystectomy with pelvic lymph node dissection and urinary diversion. This study was approved by the University of Pennsylvania's Institutional Review Board, and all patients signed informed consent.

\section{Study evaluations}

Clinical and radiologic assessment, including detailed medical history, review of concomitant medications, physical examination, laboratory testing, evaluation of cardiac function, and disease imaging (computed tomography [CT] or magnetic resonance imaging of abdomen/pelvis, CT chest or chest $\mathrm{X}$-ray) was performed at baseline and upon completion of chemotherapy. A complete blood count (CBC) and comprehensive metabolic profile were performed at baseline and on day 1 of each treatment cycle. A CBC was also repeated on days 8 and 15 of each treatment cycle. Toxicity was assessed before each treatment and monitored throughout the course of therapy. Adverse events (AEs) were graded using National Cancer Institute Common Toxicity Criteria (NCI-CTC) ver. 3. Radical cystectomy pathology was reviewed per institutional protocol, and a postchemotherapy pTpN stage was assigned using the 1997 American Joint Committee on Cancer (AJCC) staging classification system. Patients were evaluated in follow-up for ongoing toxicity, disease recurrence, and disease-specific survival, and OS. Post-treatment follow-up consisted of clinical and laboratory assessment every 3 months, as well as a chest X-ray and cross-sectional imaging of the abdomen and pelvis every 6 months.

\section{Statistical analyses}

This study was initially designed as a phase II efficacy study utilizing a Simon 2-stage optimal design with a primary end point of pT0 rate, defined as no evidence of residual disease based on pathological review of the surgical specimen. The study was designed to detect an improvement in the pT0 rate from the historical rate of $30 \%$ with gemcitabine/cisplatin neoadjuvant therapy to the alternative hypothesis of a $50 \%$ pT0 rate with $90 \%$ power and a type I error rate of 0.10. A target accrual of 46 patients was established, with 22 evaluable patients in the first stage of the study. If at least eight of these 22 patients demonstrated a pT0 response, then an additional 24 evaluable patients would be enrolled. Otherwise, the therapy would be declared ineffective, and the trial would stop. If at least 18 of the 46 total enrolled patients demonstrated a pT0 response, then GCL would be considered worthy of further investigation. The probability of early termination under the null hypothesis (pT0 rate of $\leq 30 \%$ ) was $67 \%$. Additional early stopping rules for excessive toxicity were included.

Secondary objectives were to determine the toxicities of the neoadjuvant regimen, estimate progression-free survival (PFS) and OS using the Kaplan-Meier method, and to perform exploratory correlative analyses testing the association between EGFR and HER-2 status and pT0 response at cystectomy. Analyses were based on an intent-to-treat design.

\section{Results}

\section{Patient characteristics}

Between December 2008 and July 2011, only six patients with MIBC were accrued and treated. Demographic and patient characteristics are shown in Table 1. Four men and two women were treated. The mean age of patients at the time of study enrollment was 61 years. The results of EGFR and HER-2 IHC assays are shown in Table 2. Five patients overexpressed EGFR, three patients overexpressed HER-2, and three patients overexpressed both EGFR and HER-2.

\section{Treatment exposure and toxicities}

Only three patients (50\%) completed four full cycles of GC. Three patients were switched from cisplatin to carboplatin due to renal toxicity $(n=2)$ or severe nausea/vomiting $(n=1)$. The first four patients received the protocol-specified dose of lapatinib 1,000 mg daily. Only one patient completed four cycles of lapatinib at this full dose. Dose reduction or discontinuation was required in the other three patients at this dose level. Due to toxicity concerns, the final two patients received a reduced starting dose of lapatinib of $750 \mathrm{mg}$ daily. One patient completed four full cycles of lapatinib $750 \mathrm{mg}$ daily; however, the other patient at this dose level discontinued therapy due to toxicity. All six patients proceeded to surgical resection, and no excess surgical complications were noted.

Toxicity information is summarized in Table 3. Grade 3-4 toxicities included: diarrhea $(\mathrm{n}=2)$, nausea/vomiting $(\mathrm{n}=2)$, and thrombocytopenia $(\mathrm{n}=2)$. Grade 2-3 renal toxicity was observed in two patients, requiring a switch from cisplatin to carboplatin. Two serious AEs were reported. The first was an inpatient hospitalization for fever that was considered unrelated to study treatment. The second was an inpatient 
Table 1. Patient demographics and clinical characteristics

\begin{tabular}{|c|c|c|c|c|c|c|c|c|c|}
\hline $\begin{array}{l}\text { Patient } \\
\text { No. }\end{array}$ & $\begin{array}{l}\text { Age } \\
(y r)\end{array}$ & Sex & $\begin{array}{l}\text { ECOG } \\
\text { PS }\end{array}$ & Comorbidity & $\begin{array}{c}\text { Tobacco } \\
\text { exposure }^{\text {a) }}\end{array}$ & $\begin{array}{l}\text { Clinical } \\
\text { stage }^{\text {b) }}\end{array}$ & $\begin{array}{c}\text { Presence of } \\
\text { hydronephrosis }\end{array}$ & $\begin{array}{l}\text { Serum creatinine } \\
(\mathrm{mg} / \mathrm{dL})\end{array}$ & $\begin{array}{c}\text { Hemoglobin } \\
\text { (g/dL) }\end{array}$ \\
\hline 1 & 57 & $\mathrm{~F}$ & 0 & $\begin{array}{l}\text { Hypertension, } \\
\text { hyperlipidemia, } \\
\text { osteoarthritis }\end{array}$ & $\begin{array}{l}\text { Former } \\
\text { (60 pack years) }\end{array}$ & T4 N0 & Yes & 0.86 & 9.9 \\
\hline 2 & 65 & M & 0 & $\begin{array}{l}\text { Hypertension, } \\
\text { transient ischemic attack, } \\
\text { hyperlipidemia }\end{array}$ & $\begin{array}{l}\text { Former } \\
\text { (50 pack years) }\end{array}$ & T4 N0 & Yes & 0.97 & 13.7 \\
\hline 3 & 48 & M & 0 & $\begin{array}{l}\text { Hypertension, } \\
\text { hyperlipidemia }\end{array}$ & $\begin{array}{l}\text { Current } \\
\text { (30 pack years) }\end{array}$ & T3 N0 & No & 0.94 & 14.8 \\
\hline 4 & 65 & M & 0 & Hypertension & $\begin{array}{l}\text { Former } \\
\text { (20 pack years) }\end{array}$ & T3 N0 & Yes & 0.80 & 14.5 \\
\hline 5 & 60 & $\mathrm{~F}$ & 0 & $\begin{array}{l}\text { COPD, asthma, } \\
\text { hypertension, } \\
\text { hyperlipidemia, } \\
\text { DVT }\end{array}$ & $\begin{array}{l}\text { Former } \\
\text { (30 pack years) }\end{array}$ & T2 N0 & No & 1.08 & 11.6 \\
\hline 6 & 68 & M & 0 & $\begin{array}{l}\text { Hypertension, } \\
\text { hyperlipidemia }\end{array}$ & Former & T3 N0 & Yes & 1.48 & 13.2 \\
\hline
\end{tabular}

ECOG PS, Eastern Oncology Cooperative Group performance status; F, female; M, male; COPD, chronic obstructive pulmonary disease; DVT, deep venous thrombosis. a)Pack-years=number of packs / day $\times$ number of years tobacco exposure,

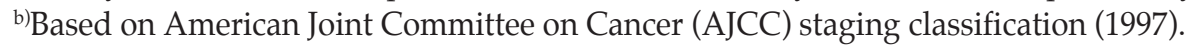

Table 2. Tumor receptor status

\begin{tabular}{ll} 
Receptor status & No. $(\%)$ \\
\hline EGFR + /HER-2 - & $2(33)$ \\
EGFR + /HER-2 + & $3(50)$ \\
EGFR - /HER-2 - & $1(17)$ \\
EGFR - /HER-2 + & 0 \\
\hline
\end{tabular}

EGFR, epidermal growth factor receptors 1; HER-2, epidermal growth factor receptor 2 .

hospitalization for diarrhea and dehydration that was deemed probably related to study treatment. There were no treatment related deaths. As the toxicity stopping rule was exceeded within the first six accrued patients, this study was reviewed with the study medical monitor and terminated early.

\section{Clinical efficacy}

Only one of six patients $(17 \%)$ achieved the primary end point of this study of a pT0 response following neoadjuvant GCL (Table 4). One additional patient had organ-confined disease (pT2). Postcystectomy pathology demonstrated nonorgan confined disease in four patients $(67 \%)$ (pT3 or pT4), and three patients $(50 \%)$ had pathologic lymph node involvement. Three patients developed disease progression at 5, 7, and 8 months following the initiation of therapy, respectively. Two of these patients died of disease. The remaining three patients, including the two patients with pT0 and pT2 surgical pathology, were alive with no evidence of disease at 14,16 , and 24 months after the initiation of therapy. Due to early termination of the study, follow-up for the estimation of PFS and OS was discontinued.

\section{Discussion}

To the best of our knowledge, this is the first clinical report on the use of lapatinib in combination with cisplatin-based chemotherapy as neoadjuvant therapy in MIBC. The addition of lapatinib to standard neoadjuvant GC was poorly tolerated, resulting in dose reduction or discontinuation of therapy in the majority of treated patients. The observed dose-limiting toxicities included those typically associated with lapatinib (diarrhea), as well as toxicities commonly associated with GC (nausea/vomiting, thrombocytopenia, and renal injury). Given the limited number of treated patients, conclusions concerning whether lapatinib resulted 
Table 3. Treatment toxicities ${ }^{\text {a) }}$

\begin{tabular}{lcccc} 
Toxicity & Grade $^{\text {b) }}$ & Grade 2 & Grade 3 & Grade 4 \\
\hline Diarrhea & 1 & - & 2 & - \\
Nausea/Vomiting & 3 & - & 2 & - \\
Fatigue & 1 & 4 & - & - \\
ANC & 1 & 2 & - & - \\
Rash & 3 & 1 & - & - \\
Thrombocytopenia & - & 2 & 1 & 1 \\
Mucositis & 1 & - & - & - \\
Renal & 1 & 1 & 1 & - \\
\hline
\end{tabular}

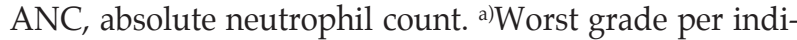
vidual patient report, ${ }^{\mathrm{b}}$ Grade assigned per National Cancer Institute Common Toxicity Criteria, ver. 3.

in increased GC toxicities or vice versa cannot be clearly stated. In addition, due to the small sample size we cannot make conclusions regarding the observed pT0 rate of $17 \%$ compared to historical controls. Furthermore, while all enrolled patients had clinical N0 disease, it is noteworthy that pathologic node involvement was demonstrated in three patients following surgery. In all cases, repeat imaging performed in the immediate preoperative period showed no clinical evidence of regional nodal metastases. In addition, the clinical understaging of MIBC is a commonly observed phenomenon. Therefore, while it is likely that these findings represent subclinical nodal metastases discovered upon lymph node dissection, we cannot exclude the possibility of disease progression through the GCL neoadjuvant regimen.

The suggested dose range for lapatinib in phase II trials is $1,250-1,500 \mathrm{mg}$ daily $[13,14]$, and multiple clinical trials of lapatinib in combination with chemotherapy have been conducted with daily lapatinib doses of 1,250-1,500 $\mathrm{mg}[15,16]$. In addition, lapatinib at daily doses of $750 \mathrm{mg}, 1,000 \mathrm{mg}$, and $1,250 \mathrm{mg}$ was studied in combination with standard-dose GC chemotherapy on a 28-day cycle length in a phase I study of advanced / metastatic bladder cancer patients [17]. This dosefinding study yielded a maximum tolerated lapatinib dose of $1,250 \mathrm{mg}$ daily [17]. Although we selected a conservative lapatinib starting dose of 1,000 mg daily for the neoadjuvant GCL regimen, toxicities nonetheless proved to be treatmentlimiting. Indeed, even with a lower starting dose of lapatinib $750 \mathrm{mg}$ daily, one of two patients discontinued therapy due to excessive toxicity.

The reason for the discrepant toxicity findings between the reported phase I GCL study in metastatic disease and the current neoadjuvant GCL study is unclear. However, the limited number of treated patients and/or differences in the treatment schedule (21-day vs. 28-day cycle length) may have contributed to the observed differences. In addition, as the trials were conducted in different geographic locations (Europe vs. United States), regional or site-specific differences may have accounted for the observed differences. It is not likely that underlying patient comorbidities significantly influenced the tolerance of this neoadjuvant regimen, as all enrolled subjects demonstrated good functional status, adequate organ function, and baseline comorbidities common to the general bladder cancer population (tobacco exposure, hypertension, and hyperlipidemia).

These results again demonstrate the limitations of determining the maximum tolerated dose in novel targeted combination regimens due to the development of cumulative toxicities after multiple cycles of therapy, as has been previously described $[18,19]$. In particular, the late toxicities (occurring after cycle 1) of molecularly-targeted agents may not be adequately reflected in the conventionally-defined recommended phase II dose $[18,20]$. These current findings amount to a negative phase I study, and clearly demonstrate the critical importance of more complete GCL dose-escalation data before further study of this regimen in the neoadjuvant setting.

Urothelial cancer is regarded as a chemosensitive disease, and neoadjuvant chemotherapy for MIBC historically yields pT0 rates of $25 \%-35 \%[2,21]$, and a pT0 response has been associated with a durable survival benefit. However, there is an increasing general belief that further alterations in conventional cytotoxic chemotherapy will not dramatically improve important clinical outcomes in urothelial cancer. Based on a recently increased understanding of the molecular biology of urothelial cancer, the addition of targeted agents to cisplatin-based neoadjuvant chemotherapy is therefore a logical next step in an effort to improve pT0 rates and survival outcomes in MIBC. However, given the potential for poor drug tolerance and the conceivable resultant risk of patients receiving inadequate standard treatment, a thorough evaluation of the maximum tolerated combination therapy dose in the advanced disease setting is prudent prior to study in the curative-intent setting.

Dual-inhibition of EGFR and HER-2 with lapatinib monotherapy has been studied in the setting of metastatic urothelial cancer (mUC) with modest results. In a phase II study of lapatinib monotherapy as second-line treatment for mUC patients, lapatinib therapy did not meet the primary endpoint of an objective response rate of $>10 \%$ [9]. However, an OS benefit was noted for patients whose tumors overexpressed EGFR and / or HER-2 ( $\geq 2+$ expression by IHC) [9]. In a histology-independent randomized discontinuation study of lapatinib monotherapy in HER-2 amplified tumors, including nine patients with advanced bladder cancer, the objective response rate was $3 \%$, and $28 \%$ of patients achieved stable disease (including three bladder cancer patients) [22]. This study was terminated early due to the low response rate 
Table 4. Pathologic outcomes at radical cystectomy

\begin{tabular}{|c|c|c|c|}
\hline Variable & No. $(\%)$ & Clinical stage $^{\mathrm{a})}$ & 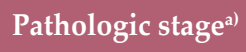 \\
\hline \multicolumn{4}{|l|}{ Surgical pathologya) } \\
\hline Organ confined disease & $2(33)$ & - & - \\
\hline pT0 & $1(17)$ & - & - \\
\hline pTis-pT1 & 0 & - & - \\
\hline pT2 & $1(17)$ & - & - \\
\hline Non-organ confined disease & $4(67)$ & - & - \\
\hline pT3-4 & $4(67)$ & - & - \\
\hline $\mathrm{pN}+$ & $3(50)$ & - & - \\
\hline \multicolumn{4}{|c|}{ Individual patient pathologic outcomes } \\
\hline Patient 1 & - & T4 N0 & T4 N2 \\
\hline Patient 2 & - & T4 N0 & T4 N2 \\
\hline Patient 3 & - & T3 N0 & T2a N0 \\
\hline Patient 4 & - & T3 N0 & T3b N2 \\
\hline Patient 5 & - & T2 N0 & T3b N0 \\
\hline Patient 6 & - & T3 N0 & T0 N0 \\
\hline
\end{tabular}

a)Based on American Joint Committee on Cancer (AJCC) staging classification (1997).

and poor accrual. More recently, a phase II/ III study of more than 200 patients with HER-1 or HER-2-positive mUC evaluated maintenance lapatinib following first-line chemotherapy. Maintenance lapatinib failed to improve PFS in this molecularly-defined population [23].

Several other therapies targeting EGFR and/or HER-2 have similarly been evaluated in the treatment of urothelial cancer. In a phase II study of 20 unselected patients with MIBC, neoadjuvant therapy with the EGFR inhibitor erlotinib was well-tolerated and yielded a pT0 rate of $25 \%$ [24]. A multicenter phase II study evaluated trastuzumab, the humanized monoclonal antibody targeting HER-2, in combination with paclitaxel, carboplatin, and gemcitabine in the treatment of patients with HER-2 overexpressing advanced urothelial cancers [25]. An overall response rate of $70 \%$ was demonstrated among 44 evaluable patients using this combination regimen [25]. Finally, recruitment for a phase II study of the EGFR and HER-2 inhibitor, afatinib, in patients with advanced urothelial cancers refractory to platinum-based chemotherapy is currently underway (NCT 02122172). Therefore, while results with lapatinib have thus far been discouraging, the targeting of EGFR and/ or HER-2 remains an area of active investigation in the treatment of urothelial malignancy.

\section{Conclusion}

In this preliminary experience, the addition of the selected dose of lapatinib to standard GC neoadjuvant chemotherapy was not successful due to excessive toxicity. The clinical efficacy of the neoadjuvant GCL regimen cannot be determined due to the small patient sample size. The results of future and ongoing studies are needed to determine whether dual-inhibition of EGFR and HER-2 in combination with cisplatin-based chemotherapy is a safe and effective therapeutic strategy in MIBC. In addition, as oncology drug development continues its shift towards molecularly-targeted therapies, numerous clinical trials combining targeted therapies with conventional cytotoxic chemotherapy will undoubtedly be conducted. With this growing enthusiasm and mounting pressure for rapid drug development, the current findings illustrate the importance of careful step-wise drug development with thorough dose-finding for novel targeted therapies.

\section{Conflicts of Interest}

This trial was funded in part by a grant from GlaxoSmithKline. 


\section{References}

1. Stein JP, Skinner DG. Radical cystectomy for invasive bladder cancer: long-term results of a standard procedure. World J Urol. 2006;24:296-304.

2. Grossman HB, Natale RB, Tangen CM, Speights VO, Vogelzang NJ, Trump DL, et al. Neoadjuvant chemotherapy plus cystectomy compared with cystectomy alone for locally advanced bladder cancer. N Engl J Med. 2003;349:859-66.

3. Advanced Bladder Cancer (ABC) Meta-analysis Collaboration. Neoadjuvant chemotherapy in invasive bladder cancer: update of a systematic review and meta-analysis of individual patient data advanced bladder cancer (ABC) meta-analysis collaboration. Eur Urol. 2005;48:202-5.

4. National Comprehensive Cancer Network. NCCN guidelines: bladder cancer [Internet]. Fort Washington, PA: National Comprehensive Cancer Network; 2015 [cited 2015 Oct 1]. Available from: http://www.nccn.org/professionals/physician_gls/f_guidelines.asp\#bladder.

5. von der Maase H, Hansen SW, Roberts JT, Dogliotti L, Oliver $\mathrm{T}$, Moore MJ, et al. Gemcitabine and cisplatin versus methotrexate, vinblastine, doxorubicin, and cisplatin in advanced or metastatic bladder cancer: results of a large, randomized, multinational, multicenter, phase III study. J Clin Oncol. 2000;18:3068-77.

6. Apolo AB, Kim JW, Bochner BH, Steinberg SM, Bajorin DF, Kelly WK, et al. Examining the management of muscle-invasive bladder cancer by medical oncologists in the United States. Urol Oncol. 2014;32:637-44.

7. Bellmunt J, Hussain M, Dinney CP. Novel approaches with targeted therapies in bladder cancer. Therapy of bladder cancer by blockade of the epidermal growth factor receptor family. Crit Rev Oncol Hematol. 2003;46 Suppl:S85-104.

8. Chow NH, Chan SH, Tzai TS, Ho CL, Liu HS. Expression profiles of ErbB family receptors and prognosis in primary transitional cell carcinoma of the urinary bladder. Clin Cancer Res. 2001;7:1957-62.

9. Wulfing C, Machiels JP, Richel DJ, Grimm MO, Treiber U, De Groot MR, et al. A single-arm, multicenter, open-label phase 2 study of lapatinib as the second-line treatment of patients with locally advanced or metastatic transitional cell carcinoma. Cancer. 2009;115:2881-90.

10. Xia W, Mullin RJ, Keith BR, Liu LH, Ma H, Rusnak DW, et al. Anti-tumor activity of GW572016: a dual tyrosine kinase inhibitor blocks EGF activation of EGFR/erbB2 and downstream Erk1/2 and AKT pathways. Oncogene. 2002;21: 6255-63.

11. Opdam FL, Guchelaar HJ, Beijnen JH, Schellens JH. Lapatinib for advanced or metastatic breast cancer. Oncologist. 2012; 17:536-42.

12. McHugh LA, Kriajevska M, Mellon JK, Griffiths TR. Combined treatment of bladder cancer cell lines with lapatinib and varying chemotherapy regimens: evidence of schedule-dependent synergy. Urology. 2007;69:390-4.

13. Versola M, Burris HA, Jones S, Wilding G, Taylor C, Pandite L, et al. Clinical activity of GW572016 in EGF10003 in patients with solid tumors. J Clin Oncol. 2004;22(14 Suppl):Abstr 3047. 14. Burris HA 3rd, Hurwitz HI, Dees EC, Dowlati A, Blackwell KL, O'Neil B, et al. Phase I safety, pharmacokinetics, and clinical activity study of lapatinib (GW572016), a reversible dual inhibitor of epidermal growth factor receptor tyrosine kinases, in heavily pretreated patients with metastatic carcinomas. J Clin Oncol. 2005;23:5305-13.

15. LoRusso PM, Jones SF, Koch KM, Arya N, Fleming RA, Loftiss J, et al. Phase I and pharmacokinetic study of lapatinib and docetaxel in patients with advanced cancer. J Clin Oncol. 2008; 26:3051-6.

16. Siegel-Lakhai WS, Beijnen JH, Vervenne WL, Boot H, Keessen M, Versola M, et al. Phase I pharmacokinetic study of the safety and tolerability of lapatinib (GW572016) in combination with oxaliplatin/fluorouracil/leucovorin (FOLFOX4) in patients with solid tumors. Clin Cancer Res. 2007;13(15 Pt 1): 4495-502.

17. Daugaard G, Sengelov L, Agerbaek M, Sternberg CN, Van Herpen C, Collette $S$, et al. Phase I results from a study of lapatinib with gemcitabine and cisplatin (GC) in advanced/ metastatic bladder cancer. J Clin Oncol. 2013;31(Suppl 6): Abstr 252.

18. Postel-Vinay S, Gomez-Roca C, Molife LR, Anghan B, Levy A, Judson I, et al. Phase I trials of molecularly targeted agents: should we pay more attention to late toxicities? J Clin Oncol. 2011;29:1728-35.

19. Galsky MD, Hahn NM, Powles T, Hellerstedt BA, Lerner SP, Gardner TA, et al. Gemcitabine, Cisplatin, and sunitinib for metastatic urothelial carcinoma and as preoperative therapy for muscle-invasive bladder cancer. Clin Genitourin Cancer. 2013;11:175-81.

20. Postel-Vinay S, Collette L, Paoletti X, Rizzo E, Massard C, Olmos D, et al. Towards new methods for the determination of dose limiting toxicities and the assessment of the recommended dose for further studies of molecularly targeted agents: Dose-Limiting Toxicity and Toxicity Assessment Recommendation Group for Early Trials of Targeted therapies, an European Organisation for Research and Treatment of Cancer-led study. Eur J Cancer. 2014;50:2040-9.

21. Dash A, Pettus JA 4th, Herr HW, Bochner BH, Dalbagni G, Donat SM, et al. A role for neoadjuvant gemcitabine plus cisplatin in muscle-invasive urothelial carcinoma of the bladder: a retrospective experience. Cancer. 2008;113:2471-7.

22. Galsky MD, Von Hoff DD, Neubauer M, Anderson T, Fleming M, Nagarwala Y, et al. Target-specific, histology-independent, randomized discontinuation study of lapatinib in patients with HER2-amplified solid tumors. Invest New Drugs. 2012; 30:695-701.

23. Powles T, Huddart RA, Elliott T, Jones R, Hussain SA, Crabb SJ, et al. A phase II/III, double-blind, randomized trial comparing maintenance lapatinib versus placebo after first line chemotherapy in HER1/2 positive metastatic bladder cancer patients. J Clin Oncol. 2015;33(Suppl):Abstr 4505.

24. Pruthi RS, Nielsen M, Heathcote S, Wallen EM, Rathmell WK, 
Godley P, et al. A phase II trial of neoadjuvant erlotinib in patients with muscle-invasive bladder cancer undergoing radical cystectomy: clinical and pathological results. BJU Int. 2010;106:349-54.

25. Hussain MH, MacVicar GR, Petrylak DP, Dunn RL, Vaisham- payan U, Lara PN Jr, et al. Trastuzumab, paclitaxel, carboplatin, and gemcitabine in advanced human epidermal growth factor receptor-2/neu-positive urothelial carcinoma: results of a multicenter phase II National Cancer Institute trial. J Clin Oncol. 2007;25:2218-24. 